\title{
An in vitro method to select malignant cells from surgical biopsies of breast cancer patients
}

\author{
Kelly E. Corcoran ${ }^{1}$, Prem S. Patel ${ }^{2}$, and Pranela Rameshwar ${ }^{3 *}$ \\ ${ }^{1}$ Graduate School of Biomedical Sciences, Interdisciplinary Program, UMDNJ-Newark Campus. \\ 2Brookdale University Hospital and Medical Center, Department of Surgery, Brooklyn, NY. \\ ${ }^{3}$ Department of Medicine, UMDNJ-New Jersey Medical School, Newark, NJ.
}

*Corresponding Author: Pranela Rameshwar, Ph.D., UMDNJ-New Jersey Medical School, MSB, Rm. E-579, 185 South Orange Ave, Newark, NJ 07103 Phone: (973) 972 0625; Fax: (973) 972 8854; Email: rameshwa@umdnj.edu

Submitted: December 16, 2004; Revised: January 7, 2005; Accepted: January 10, 2005.

Indexing terms: Bone marrow; Cultured, cells; Breast neoplasms.

Abbreviations: SDF-1 $\alpha$ : Stromal cell-derived factor $1 \alpha$.

\section{ABSTRACT}

To date, breast cancer (BC) research is mainly studied with cell lines. These cells were passaged multiple times, acquiring phenotypes, additional mutations and epigenetic changes. These changes make the passaged cell lines different from the original malignancy. Thus cell lines, although useful as models could be improved with additional studies with primary BC. It is difficult to obtain malignant cells from breast tissues without contamination from surrounding healthy cells. Selection and expansion of malignant cells from surgical tissues have proved to be daunting tasks. This study describes a reliable and reproducible method for isolating and expanding malignant cells from surgical breast tissues. The method uses co-cultures with BM stroma to select for the cancer cells while the healthy cells undergo rapid cell death. Studies are described to show the cloning efficiencies and sensitivity of the method using surgical samples of varying sizes, different stages of $\mathrm{BC}$, and samples from needle biopsies.

\section{INTRODUCTION}

Breast cancer $(\mathrm{BC})$ metastasis to the bone marrow $(\mathrm{BM})$ correlates with poor prognosis (1). Seemingly curative therapies in patients with both metastatic and nonmetastatic $\mathrm{BC}$ have recurrence of $\mathrm{BC}$ cells from the $\mathrm{BM}$ (1). Such resurgence could occur even twenty years after remission $(1,2)$. Based on these reports, it is hypothesized that $\mathrm{BC}$ cells (BCCs) entering and surviving in BM are either subsets of BCCs with unique properties, or they are located in an area to protect them from current treatment modalities. The ability of BCCs resurging from the $\mathrm{BM}$ is consistent with preference for this organ (2). Since similar resurgence occurred even in cases with early stages of BC it appears BCCs might have entered BM long before the tumor could be detected by current clinical methods.

BM toxicity limits the dose of current cancer therapies (3). Thus, if a special type of BCC is within specialized region of the $\mathrm{BM}$, then targeting $\mathrm{BCC}$ in $\mathrm{BM}$ would be a clinical challenge. To mimic in vivo behavior of BCCs, it is essential to use primary BCCs in experiments since these cells would have limited divergence as might occur with cell lines.

Studies using BC cell lines with varying degrees of metastatic potentials are models in the majority of laboratories. Use of cell lines is convenient due to their availability through tissue depository banks, and 
simplicity with regards to culture maintenance. Some cell lines have been distributed within laboratories so that relevant information regarding their source and number of passages are lost. This could lead to heterogeneity within a particular line and lead to seemingly irreproducible data among laboratories. In summary, BC cell lines might not show consistent results, and might not accurately represent the behavior of cancer cells in vivo.

Another disadvantage for BCCs subjected to multiple passages relates to their genomic instability resulting in a population of cells with varying degrees of tumorigenic potential. It is advisable to verify studies from cell lines with primary BCCs. Another limitation when primary BCC are used pertains to the size of surgical tissues. For small samples, clinical analyses have first preference leaving no sample for experimental analyses. These limits are evident when samples are obtained by noninvasive methods such as needle biopsies. Another major disadvantage is difficulty in separating malignant and normal cells in surgical tissues.

Studies from our laboratory reported a novel method to isolate primary BCCs in co-cultures with BM stroma. The method has been proven effective and sensitive even for needle biopsies. The co-culture method is also applied in studies to understand the behavior of BCCs within BM. Methods to use primary BCCs is underscored by recent findings on SDF- $\alpha-C X C R 4$ interactions in BC metastasis (4). Malignant breast tissue, unlike healthy tissue show high expression of CXCR-4. Since stromal cells produce SDF-1 $\alpha$ (8), BCCs expressing CXCR4 could be attracted and retained to organs that express SDF-1, such as BM, lung, and liver (4). The density of CXCR-4 on BCC is proportional to the invasiveness of the cancer (5). Recent studies with mice show that there might be two subtypes of BCC that enter the BM (6). The aforementioned examples on SDF-1-CXCR4 argue for studies with primary BCCs to understand the fine-tuned mechanisms facilitating $\mathrm{BC}$ metastasis to the $\mathrm{BM}$, and late stage resurgence in $\mathrm{BM}$.

Previous studies show BCCs with high proliferative potential is found within the central/cellular areas of the BM cavity (6). Another BC subset with long doubling times is located close to the endosteum (6). Defining the properties of BCC subtypes in the BM and understanding the mechanisms involved in BCC entry in the $\mathrm{BM}$ are keys to improve cancer treatment and eradication. The method to isolate malignant cells will provide the opportunity to compare BCCs at different stages of the cancer, and also those that enter the BM at different periods during the course of the disease. To identify the cells entering early in different organ could lead to understandings on the mechanisms of entry and the signature of these cells, leading to cancer prevention, partly by early detection.

\section{MATERIALS AND METHODS}

\section{Reagents and antibodies}

$\alpha$-Minimum Essential Medium ( $\alpha$-MEM), glutamine and hydrocortisone were purchased from Sigma (St. Louis, MO). Fetal Calf sera (FCS) and Horse Sera (HS) were purchased from Hyclone Laboratories (Logan, UT). PEcytokeratin $\mathrm{mAb}, \mathrm{PE}$ - rat anti mouse kappa and PE$\mathrm{CD} 14 \mathrm{mAb}$ were purchased from BD Bioscience (San Jose, CA). Prolyl-4-hydroxylase $\mathrm{mAb}$ was purchased from Dako (Glostrup, Denmark). Anti-Epithelial and anti-fibroblast were purchased from Miltenyi Biotec, Auburn, CA) respectively.

\section{Cell lines}

The following cell lines were purchased from American Type Culture Collection (Manassas, VA): Tumorigenic cell lines, T47D and MDA-MB-330 and non-tumorigenic cell lines, MCF12A and MCF 10A.

\section{Primary breast tissue}

Excess tissues were taken from samples of surgical procedures. The surgical interventions were done in patients diagnosed with different stages of BC. At the time of surgery patients were not on any medication. Samples were provided from two sources. The major source of breast tissue was from Brookdale Hospital (Brooklyn, NY). Use of breast tissues followed a protocol approved by the institutional review board of University of Medicine and Dentistry of New Jersey (Newark, NJ) and Brookdale Hospital (Brooklyn, NY). Patient samples were also provided by the Cooperative Human Tissue Network. Table 1 shows representative subsets of patients' profile of tissues. Excess samples from needle biopsies were also obtained from Brookdale Hospital. 
Table 1: Single cell suspensions were prepared from surgical tissues of different stages of BC.

\begin{tabular}{lccc}
\hline & \multicolumn{3}{c}{ Stage of BC } \\
& $\mathbf{M ~ 0}$ & $\mathbf{M ~ I}$ & $\mathbf{M}$ III \\
Starting population & $1012 \pm 50$ & $5180 \pm 32$ & $\mathbf{n}=\mathbf{1 0}$ \\
Total cells at P4 & $2 \times 10^{6} \pm 55$ & $6 \times 10^{6} \pm 90$ & $5 \times 10^{6} \pm 66$ \\
Fold expansion & $2.0 \times 10^{3}$ & $1.2 \times 10^{3}$ & $1.2 \times 10^{3}$ \\
Colonies $/ \mathbf{1 0}^{3}$ & $820 \pm 23$ & $744 \pm 14$ & $868 \pm 15$ \\
Cloning efficiencies & $82 \%$ & $74 \%$ & $87 \%$ \\
\hline
\end{tabular}

Fibroblasts were depleted from surgical breast tissues. Cells retrieved (starting population) were placed in co-cultures with BM stroma. At confluence, cytokeratin positive cells were selected and then placed in multiple passages of co-cultures with fresh BM stroma. The mean \pm SD of total cytokeratin positive cells are shown for passage 4 (P4). At P4 cells were studied in clonogenic assays.

\section{Cell suspension from surgical breast tissues}

Cells (normal and malignant) were retrieved from the breast tissues using the following two methods: a) flushing with a 1-cc syringe containing any type of cell culture media; and b) dislodging cells with serrated-end forceps. Fibroblasts were removed using double positive selection with anti-fibroblast microbeads. Depletion of fibroblasts was verified by flow cytometry with FITCanti-fibroblasts and PE-anti-cytokeratin. Viability of selected cells was $>95 \%$, by trypan blue exclusion. Biopsies from three different patients with stage $0 \mathrm{BC}$ (Table 1) were obtained and also cleared of fibroblasts (as above). Fibroblast-depleted cells from patients with BC are hereafter referred as breast cells because the cells might contain both malignant and normal cells. After coculture and selection of anchorage independent growth, the cells were referred as primary BCCs (6). The cells were passaged at least four times before use in assays. Cryopreservation up to two years did not alter their anchorage-independent properties, nor their ability to form co-cultures.

\section{Preparation of BM stroma}

$\mathrm{BM}$ aspirate, $10^{7}$ nucleated cells from healthy donors between ages 18-25 years were added to $25 \mathrm{~cm}^{2}$ tissue culture flasks (Falcon 3109). After selection, stromal cells were resuspended in stromal culture medium II, consisting $\alpha$-MEM with $20 \%$ FCS. Plates were incubated 3 days at $37^{\circ} \mathrm{C}$; mononuclear cells were separated by Ficoll-Hypaque density gradient and then replated in fresh medium. Cells were incubated until confluency, approximately 3 weeks. During this time, cultures were fed weekly by replacing fifty percent of the culture medium with fresh medium. At confluence trypsinsensitive, adherent cells were passed at least five times. Flow cytometry studies indicate stromal cells were CD14 $(-)$ and prolyl 4-hydroxylase (+).

\section{Co-cultures with established cell lines}

BCCs refer to both cell lines and primary cells. Equivalent numbers of BCCs and BM stromal were cocultured in stromal growth media with weekly replacement of $50 \%$ of the culture media. For cultures in $25 \mathrm{~cm}^{2}$ tissue culture flasks, cultures were initiated with $10^{2}$ cells of each cell subset. To perform timeline studies, at different times, cells are trypsinized and the BCCs are positively selected by subjecting the cells twice to positive selection with Dynabead conjugated anticytokeratin, as described (7). Both stromal and BCC cell populations are counted and the purity of each were verified by flow cytometry for epithelial and fibroblasts antibodies. For epithelial, cells were labeled with PEcytokeratin $\mathrm{mAb}$. Indirect labeling was done for stroma, first with prolyl 4-hydroxylase $\mathrm{mAb}$ followed with $\mathrm{PE}$ rat anti-mouse IgG. Cells separated by this isolation procedure show purity of more than ninety nine percent.

\section{Selecting BCCs from primary cultures}

Epithelial cells retrieved from surgical breast tissues, $10^{3}$ $10^{6}$, were added to $25 \mathrm{~cm}^{2}$ tissue culture flasks with $7 \mathrm{ml}$ of stromal media and stromal cells at $20-40 \%$ confluence. At confluence, the breast cells are isolated as described for co-cultures with cell lines. Cells retrieved are counted and the process repeated three times. Cells are then studied for cloning efficiency in methylcellulose matrix (see below). If the efficiency is $<90 \%$, the selection process was repeated until cloning efficiencies were $>90 \%$.

\section{Clonogenic assays}

Clonogenic assays were performed as described (6). Briefly, $10^{3}$ cells $/ \mathrm{ml}$ were resuspended in $1.2 \%$ methylcellulose containing media taken from the cocultures. One $\mathrm{ml}$ of cell suspension was added to $35 \mathrm{~mm}$ suspension dishes (Nunc, Cat\#171099) and the cultures were incubated for 1 wk at $37^{\circ} \mathrm{C}$. Colonies with $>15$ cells were counted and the cloning efficiency presented as percent of total cells placed in culture. 


\section{RESULTS AND DISCUSSION}

This study depleted cells from breast biopsies of fibroblasts rather than subjecting the cells positively with anti-epithelial cells. Although this appears to place the breast cells under unnecessary stress we have found loss of malignant cells when epithelial cells are selected. Thus, if large breast tissues are obtained from the surgeon, positive selection could be performed. However, if there are variation in surface epithelial, crucial subsets might be missed in positive selection.

Cells from breast tissues were placed in suspension and then depleted of fibroblasts. The breast cells were either cultured alone in stromal media or added to stromal cultures. Cultures in stromal media alone did not survive. However, those cultured in the presence of BM stroma continued to survive until confluent. Initial plating of co-cultures comprised BM stroma and BCCs (T47D and MDA-MB-330) or non-tumorigenic cells (MCF $10 \mathrm{~A}$ or MCF 12A). Figure $1 \mathrm{~A}$ shows $\mathrm{T} 47 \mathrm{D}$ as representative of co-cultures with BCCs. Since nontumorigenic cells did not survive in co-cultures, these experiments are not shown. Controls cultures Subsequent passage is represented in Figure 1B. By the fourth passage, the cells are able to be cultured in the absence of BM stroma (Fig. 1C). Parallel cultures with a cell line, T47D are represented in Figure 1D-1F. Cells in both $1 \mathrm{C}$ and $1 \mathrm{~F}$ were similar by morphology and by phenotype, positive for cytokeratin (not shown).
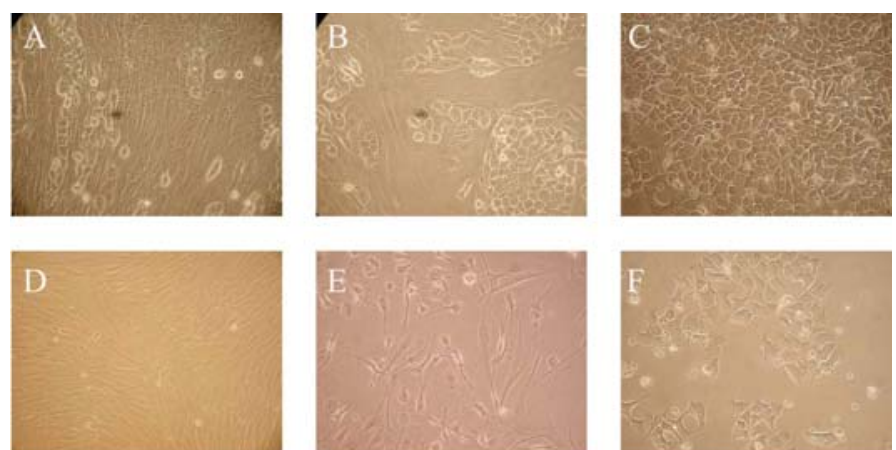

Fig. 1: $B C$ cells from patients were plated on $B M$ stroma in co-culture. Cells were passaged together four times. At passage $5, B C C$ were isolated and characterized. Parallel studies were performed using established BC lines for comparison. A) Initial co-culture of patient BCC and BM stroma. B) Patient BCC and BM stroma at P4. C) Primary BCC isolated from stroma after co-culture. D) Initial co-culture of T47D and BM stroma. E) T47D and BM stroma at P4. F) T47D isolated from stroma after co-culture. Primary cells represent studies from seven patients. Cell line studies represent studies with two different cells, each performed seven times.

The results shown in Figure 1 do not prove that the cells could be cultured in an anchorage-independent manner. To this end, cells represented in Figure $1 \mathrm{C}$ were assayed in methylcellulose matrix for clonogenic potential. To prove that anchorage-independent clones can be derived from different stages of $\mathrm{BC}$, the assays were performed with cells selected from early to late stages of BC. Table 1 describes the steps beginning from the number of cells selected from Stages M0, I and III BC patients. The results showed a cloning efficiency of $82 \%$ for the malignant cells from the Stage M0 patients, $74 \%$ stage MI, and $87 \%$ Stage MIII. This indicates that anchorage independent clones can be selected and expanded from various stages of $\mathrm{BC}$.

The co-culture method presented in this report was originally designed to select malignant cells from surgical breast tissues. The method has been expanded to select and expand malignant cells from breast biopsies. The method has been shown to be sensitive to select malignant cells from small tissue samples, including patients with Stage M0 BC. The method is also sensitive to select malignant cells from needle biopsies.

In summary, co-culture of cells from breast tissues with the stroma still resulted in the selection of the anchorage independent malignant cells when the tissues are derived from different stages of $\mathrm{BC}$ (Table 1). Co-cultures from tissues taken from Stage M0 BC patients exhibited significantly slower growth rate compared to co-cultures with cells from Stage III BC. Figure 2 shows the growth of BCCs populations for BCCs taken from patients with Stages M0 and III.

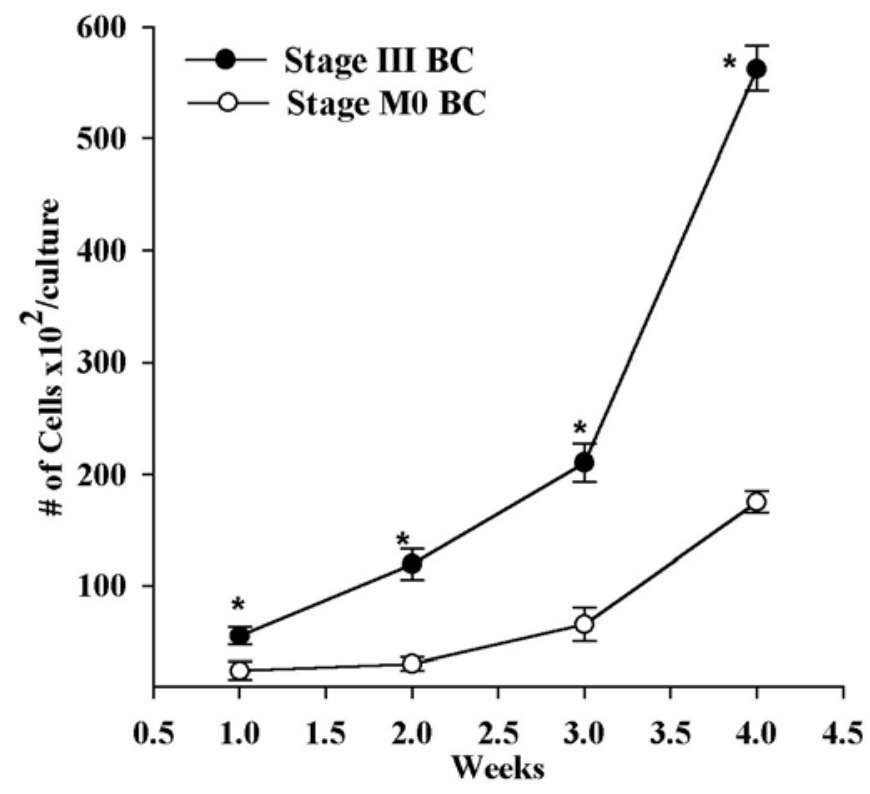

Fig. 2: Co-cultures of BM stroma and primary BCCs from Stages III and MO. At different times during co-cultures, epithelial cells were positively selected and then counted. The results are shown as mean \pm SD of seven different experiments. 
This is probably due to two reasons: 1 . The co-cultures are initiated with significantly lower number of malignant cells, 2 . The early stage of $\mathrm{BC}$ might have slower growing malignant cells. The co-culture described in this report will begin to characterize BCCs from different stages of BC. Another application for the coculture with BM stromal cells is an excellent tool for selecting malignant cells and expanding them in culture from small samples for clinical and/or research analyses. An important aspect of our model of selecting BCCs in stromal co-culture is whether the method is selecting specific malignant cells with preference for BM. The information is important in light of recent studies shown organ-specific potential of distinct BCCs (9). The method described in this report could be valuable for future research to study differences in BCCs in various sites of metastasis. Of note is that passage of the cells in the method is meant to eliminate non-tumorigenic cells since passage 1 cells are anchorage-dependent.

\section{ACKNOWLEDGMENTS}

This study was supported by CA-89868 awarded by the National Cancer Institute.

\section{REFERENCES}

1. Mansi JL, Berger U, McDonnell T, Pople A, Rayter Z, Gazet JC et al. The fate of bone marrow micrometastaes in patients with primary breast cancer. J Clin Oncol 1989; 7:445-449.

2. Gluck S. Autologous transplantation for patients with advanced breast cancer with emphasis on bony metastasis. Can J Oncol 1995; 1:58-62.
3. Moore KA, Ema $\mathrm{H}$, Lemischka IR. In vitro maintenance of highly purified, transplantable hematopoietic stem cells. Blood 1997; 89:4337-4347.

4. Muller A, Homey B, Soto H, Ge N, Catron D, Buchanan ME et al. Involvement of Chemokine Receptors in Breast Cancer Metastasis. Nature 2001; 410:50-56.

5. Kato M, Kitayama J, Kazama S, Nagawa H. Expression Pattern of CXC chemokine Receptor-4 is correlated with lymph node metastasis in human invasive ductal carcinoma. Breast Can Res 2003; 5:R144-R150.

6. Rao G, Patel PS, Idler SP, Maloof P, Gascon P, Potian JA et al. Facilitating Role of Preprotachykinin-I Gene in the Integration of Breast Cancer Cells within the Stromal Compartment of the Bone Marrow: A Model of Early Cancer Progression. Can Res 2004; 64:28742881.

7. Rameshwar P, Zhu G, Donnelly RJ, Qian J, Ge H, Goldstein KR et al. The dynamics of bone marrow stromal cells in the proliferation of multipotent hematopoietic progenitors by substance P: An understanding of the effects of a neurotransmitter on the differentiating hematopoietic stem cell. J Neuroimmunol 2001; 121:22-31.

8. Bonnet D. Haematopoietic Stem Cells. J Path 2002; 197:430-440.

9. Minn AJ, Kang Y, Serganova I, Gupta GP, Giri DD, Doubrovin $\mathrm{M}$ et al. Distinct organ-specific metastatic potential of individual breast cancer cells and primary tumors. J Clin Invest 2005; 115:44-55. 


\section{PROTOCOLS}

Perform all procedures under sterile conditions.

\section{A. Stromal isolation, 2-step procedure:}

Step 1

1. Get approval for use of human tissues by the Institutional Review Board.

2. Obtain bone marrow (BM) aspirates from healthy donors. The aspirate is obtained in a syringe containing preservative-free heparin at $50 \mathrm{U} / \mathrm{ml}$. The heparin is diluted in any tissue culture media containing $50 \mathrm{U} / \mathrm{ml}$ Penicillin and $0.05 \mathrm{mg} / \mathrm{ml}$ Streptomycin.

3. Count the nucleated cells in the aspirates.

4. Add 107 nucleated BM aspirate cells into a $25 \mathrm{~cm}^{2}$ tissue culture flasks (Falcon 3109). Adjust total volume to $7 \mathrm{ml}$ of stroma-I media.

Stroma-I Media:

- $\alpha$-MEM containing 12.5\% Fetal Bovine Serum, 12.5\% Horse Sera, 0.1\% $\mu \mathrm{M}$ Hydrocortisone, $0.1 \mu \mathrm{M} 2-$ mercaptoethanol, and $1.6 \mathrm{mM}$ glutamine;

- Incubate flasks in a $37^{\circ} \mathrm{C}$ incubator with $5 \% \mathrm{CO}_{2}$.

5. At day 3, remove the non-adherent cells from the flasks and place in a conical tissue culture tube. Combine the cells of each donor. Wash residual red blood cells (RBCs) in the flasks with sera-free media and transfer to the tubes containing the non-adherent cells. The wash media should be at room temperature.

6. Avoid the tissue culture flasks to dry by quickly adding $6 \mathrm{ml}$ of stromal media. Return flasks to the culture incubator.

7. Centrifuge tubes containing the non-adherent cells at $500 \mathrm{~g}$. If the total volume is $\sim 50 \mathrm{ml}$, centrifuge for 20-30 min. If $10-20 \mathrm{ml}$, centrifuge for 10-15 min.

8. Aspirate media.

9. Resuspend pellet in sera-free $\alpha$-MEM. Determine the total volume to resuspend the cells using the following guide: - Pellets from five flasks should be resuspended in $20 \mathrm{ml} \alpha$-MEM. If the pellet is from more than five flasks, split the cell suspension in multiple $50 \mathrm{ml}$ conical tissue culture tubes.

10. Add equal volumes of Ficoll Hypaque to the bottom of each tube. Centrifuge tubes at room temperature for 25-30 $\min$ at $500 \mathrm{~g}$.

11. Carefully remove tubes from centrifuge. Aspirate the top layer comprising the suspension media. The next layer of white represents the mononuclear fraction (BMNC). Using a Pasteur pipet aspirate the BMNC and transfer into a clean sterile conical tissue culture tube. This would require taking a small volume of Ficoll Hypaque. Resuspend the cells with 10-20 volumes of sera-free $\alpha$-MEM.

12. Centrifuge tubes at $500 \mathrm{~g}$ for $20-30 \mathrm{~min}$ at room temperature.

13. Aspirate the media. Resuspend the pellet in stroma-I media. The volume of media will depend on the total numbers of flasks from which the non-adherent cells were obtained. For example, if the starting numbers were 3 flasks, add $3 \mathrm{ml}$ of stromal media.

14. Remove the tissue culture flasks from the incubator.

15. Add $1 \mathrm{ml}$ of cell suspension/flask.

16. Re-incubate flasks. Each week replace $50 \%$ of the culture media with fresh stromal media until confluence.

\section{Step 2}

1. Trypsinize confluent stromal cells from step 1 by adding $1-2 \mathrm{ml}$ of $0.05 \%$ Trypsin with $0.053 \mathrm{Na}$-EDTA to each flask. Incubate in a $37^{\circ} \mathrm{C}$-incubator for $5 \mathrm{~min}$. Examine flasks with an inverted microscope. If the cells de-adhered, 
collect the cells and place into a conical tissue culture tube containing $\alpha$-MEM with $10 \%$ FCS. Pool cells from a particular donor. If the cells do not appear de-adhered, tap the flasks and re-examine the flasks. If the cells remain adherent, reincubated the flasks until the cells de-adhered.

2. Pellet cells by centrifuging at $500 \mathrm{~g}$ for $10-15 \mathrm{~min}$. Collect trypsin-sensitive cells and place in a test tube containing any type of tissue culture media containing $10 \%$ FCS.

3. Resuspend cells in Stroma-II media. Add $3 \mathrm{ml}$ of media for cells obtained from each flask.

Stroma II Media:

- $\quad \alpha$-MEM with $20 \%$ heat inactivated FCS.

4. Add $1 \mathrm{ml}$ of cell suspension to $25 \mathrm{~cm}^{2}$ tissue culture flask. Adjust the volume to $7 \mathrm{ml}$ with fresh Stroma-II media.

5. At cell confluence, repeat cells passages, \#1-4 of Step 2.

6. Repeat step 5 four times.

7. Confirm the purity of stromal cells by immunofluorescence using the following antibodies:

- Anti-CD14 (PE, FITC or any other fluorochrome), expected to be negative.

- Anti-fibroblasts (non-overlapping fluorochrome, i.e., different from the conjugate used for anti-CD14. For example, if anti-CD14 is conjugated to PE, anti-fibroblasts should be conjugated to any fluorochrome but PE, expected to be positive).

\section{Immunofluorescence stain}

1. Collect cells in a polypropylene test tube

2. Spin cells 10 minutes at 150rpm

3. Resuspend cell pellet in 1 XPBS to wash

4. Spin 10 minutes at $150 \mathrm{rpm}$

5. Aspirate PBS and repeat

6. Resuspend cell pellet in 1-2ml PBS

7. Add $1^{\circ}$ Antibodies (CD14 and Anti-fibroblast) 1:1000

8. Incubate 1-2 hours, shake every 10minutes

9. Wash 2 times with 1 XPBS

10. Add $300 \mu \mathrm{l}$ of PBS

11. Read on the Flow Cytometer

* If CD14 is positive, continue to passage cells since macrophages are insensitive to trypsinization.

\section{B. Isolation of epithelial cells from surgical breast tissues}

1. Obtain surgical breast tissue sample from excess patient sample. (Obtain informed consent from patients based on a protocol approved by the Institutional Review Board)

2. Flush cells from the tissue as follows:

- Forcefully passing any sera-free tissue culture media through a 1-cc syringe.

- Use a sterile serrated tip tweezers to separate the cells within the tissue samples.

3. To avoid cell clumps, place petri dishes containing cell suspension at $\sim 30-45$ degree angle. This would allow for large clumps to remain at the top. Collect the clump-free cell suspension in sterile conical tissue culture tubes. Fill the tube with any sera-free media. Pellet cells by centrifuging at $500 \mathrm{~g}$ for 10-15 min at room temperature.

4. Resuspend the cells in $\mathrm{Ca}^{++} / \mathrm{Mg}^{++}$-free PBS (pH7.2).

5. Deplete the cell suspension of fibroblasts with anti-fibroblast microbeads from Miltenyi Biotec (Cat\#130-050-601) using manufacturer's instructions. The following is a brief outline of the protocol used to deplete fibroblasts followed by positive selection of epithelial cells:

- Resuspend cells at $10^{7} / \mathrm{ml}$ in PBS. 
- Add anti-fibroblast microbeads. This step deviates from manufacturer's suggestion. Incubate at room temperature for 1-2 h. Mix at 10-min intervals. Note: The incubation time could vary based on the degree of malignancies in the biopsies. Consistently, 1-2 $\mathrm{h}$ incubation period has shown consistent results.

- Select the magnetic-coupled fibroblasts. Proceed with the negative fraction. Use an aliquot of cells and determine by immunofluorescence if the negative fraction is devoid of fibroblasts. To do this, label cells with FITC-anti-fibroblast at 1/1000 dilution final concentration. Determine non-specific labeling with FITC-isotype control. Immediately examine cells microscopically or by FACscan. If the results show the presence of fibroblasts, repeat the selection process until labeling for fibroblasts determined depletion.

6. Positively select epithelial cells using human epithelial antigen (HEA) microbeads from Miltenyi Biotec., according to manufacturer's instructions.

- Resuspend cells at $10^{7} / \mathrm{ml}$ in $1 X$ PBS

- Add anti-epithelial microbeads. Incubate at room temperature for $2 \mathrm{~h}$. Mix at 10-min intervals.

- Select the magnetic-coupled epithelial cells. Remove the negative fraction. Resuspend the positive fraction in media. Use an aliquot of cells and determine by immunofluorescence if the positive fraction is pure. To do this, label cells with FITC-anti-CD31 at 1/1000 dilution final concentration. Determine non-specific labeling with FITCisotype control. Immediately examine cells microscopically or by FACscan. If the results show the presence of endothelial cells, repeat the selection process until labeling for epithelial cells determined depletion.

- Plate epithelial cells in appropriate BCC media

\section{Co-culture of BCC and BM}

1. Add equal numbers of BM stromal cells and primary breast cells into flasks containing stromal media II.

2. Replace $50 \%$ of the media weekly until confluence.

3. Use the method above for positive selection of epithelial cells.

4. Reestablish co-cultures with fresh stroma and the selected epithelial cells.

5. Repeat steps 3 and 4 for up to four times.

6. Test the malignancies of the epithelial fraction in clonogenic assays.

\section{Clonogenic assay}

1. Add Breast cancer cells $10^{3} / \mathrm{ml}$ in $1.2 \%$ methylcellulose matrix with stromal media

2. Add $1 \mathrm{ml}$ of cell suspension in $35 \mathrm{~mm}$ Nunc suspension dishes.

3. Incubate one week at $37^{\circ} \mathrm{C}$

4. Count colonies with $>25$ cells

\section{Preparation of methylcellulose}

Note: do not use fresh methylcellulose. Freeze at least 2 month prior to use, this helps the methylcellulose to dissolve.

\section{Materials}

- $\quad$ Methylcellulose, 4000 centipose - Fisher Scientific (Cat\# M-352)

- 2 liter Erlenmeyer flask

- Large magnetic stir bar (must be large enough to stir methylcellulose at semisolid phase)

- 1.5 liters of endotoxin-free double distilled water

- Autoclavable measuring cylinder $1 \mathrm{~L}$

- Sodium bicarbonate solution (7.5\%) 


\section{Autoclave}

- $12 \mathrm{~g}(1.2 \%)$ methylcellulose (weigh in beaker then cover with double layer of aluminum foil)

- Erlenmeyer flask with stir bar

- Measuring cylinder, covered with double layer of foil

- 1.5 liters of Endotoxin free double distilled water (3-500 $\mathrm{ml}$ bottles)

(Autoclave dry and wet items separately)

\section{Methylcellulose (1.2\%)}

- Chill $1500 \mathrm{ml}$ bottle of water to $4^{\circ} \mathrm{C}$

- Prepare 2x Iscove's Media (if Iscove's is not available, use 2x $\alpha$-MEM)

- Set up a stirring/hot plate under sterile hood. Slowly pour methyl cellulose in $300 \mathrm{ml}$ boiling distilled water.

- Stir vigorously while pouring.

- Stir just under boiling until the mixture is homogenous.

- Add $200 \mathrm{ml}$ cold distilled water

- Cool to room temperature. Add $500 \mathrm{ml} 2 x$ Iscove's (room temp) while stirring

- Add $26.7 \mathrm{ml}$ sodium bicarbonate $(7.5 \%)$

- Continue stirring in the cold room for 48 hours

- Aliquot in $50 \mathrm{ml}$ tubes, store at $-20^{\circ} \mathrm{C}$

- Thaw at $4^{\circ} \mathrm{C}$ prior to use 